\title{
Host phylogeny and host ecology structure the mammalian gut microbiota at different taxonomic scales
}

\author{
Connie A. Rojas ${ }^{1,2,3^{*}}$ (D), Santiago Ramírez-Barahona ${ }^{4}$, Kay E. Holekamp ${ }^{1,2,3}$ and Kevin R. Theis ${ }^{3,5}$
}

\begin{abstract}
The gut microbiota is critical for host function. Among mammals, host phylogenetic relatedness and diet are strong drivers of gut microbiota structure, but one factor may be more influential than the other. Here, we used 16S rRNA gene sequencing to determine the relative contributions of host phylogeny and host diet in structuring the gut microbiotas of 11 herbivore species from 5 families living sympatrically in southwest Kenya. Herbivore species were classified as grazers, browsers, or mixed-feeders and dietary data (\% C4 grasses in diet) were compiled from previously published sources. We found that herbivore gut microbiotas were highly species-specific, and that host taxonomy accounted for more variation in the gut microbiota (30\%) than did host dietary guild (10\%) or sample month (8\%). Overall, similarity in the gut microbiota increased with host phylogenetic relatedness $(r=0.74)$ across the 11 species of herbivores, but among 7 closely related Bovid species, dietary \%C4 grass values more strongly predicted gut microbiota structure $(r=0.64)$. Additionally, within bovids, host dietary guild explained more of the variation in the gut microbiota (17\%) than did host species (12\%). Lastly, while we found that the gut microbiotas of herbivores residing in southwest Kenya converge with those of distinct populations of conspecifics from central Kenya, fine-scale differences in the abundances of bacterial amplicon sequence variants (ASVs) between individuals from the two regions were also observed. Overall, our findings suggest that host phylogeny and taxonomy strongly structure the gut microbiota across broad host taxonomic scales, but these gut microbiotas can be further modified by host ecology (i.e., diet, geography), especially among closely related host species.
\end{abstract}

Keywords: Herbivore gut microbiome, African mammals, Phylosymbiosis, Diet, 16S rRNA gene sequencing, Host ecology, Host geography

\section{Background}

The gut microbiota, which is the collection of microbes inhabiting the gastrointestinal tract, is essential to host functioning. In mammals, resident gut microbes promote the digestive efficiency of their hosts by synthesizing vitamins, breaking down fiber, and supplementing the host with energy released from fermentation [1-4]. The gut microbiota also interacts with the host immune

\footnotetext{
* Correspondence: rojascon@msu.edu

'Department of Integrative Biology, Michigan State University, East Lansing, $\mathrm{MI}$, USA

2Ecology, Evolution, and Behavior Program, Michigan State University, East Lansing, MI, USA

Full list of author information is available at the end of the article
}

system, and may also modulate behavior [5-7]. Due to the critical importance of the gut microbiota for host performance, research has focused on determining the forces that shape its assembly and composition. Decades of research show that across vertebrate hosts, the gut microbiota is predominantly shaped by host phylogeny and ecology. Closely related host species tend to have more similar gut microbiotas than more distantly related host species [8-12] and this congruence between host phylogenetic relatedness and gut microbiota similarity is termed "phylosymbiosis" [13-15]. However, gut microbiotas can also be further shaped by their host's ecology, including their host's diet, habitat, and geographic

(c) The Author(s). 2021 Open Access This article is licensed under a Creative Commons Attribution 4.0 International License, which permits use, sharing, adaptation, distribution and reproduction in any medium or format, as long as you give

appropriate credit to the original author(s) and the source, provide a link to the Creative Commons licence, and indicate if changes were made. The images or other third party material in this article are included in the article's Creative Commons licence, unless indicated otherwise in a credit line to the material. If material is not included in the article's Creative Commons licence and your intended use is not permitted by statutory regulation or exceeds the permitted use, you will need to obtain permission directly from the copyright holder. To view a copy of this licence, visit http://creativecommons.org/licenses/by/4.0/ 
location [16-19]. Thus, although both of these host factors may shape the gut microbiota, their relative contributions might be influenced by a variety of variables including the taxonomic breadth of the host species surveyed, and the diversity of host habitats, diets, and gut physiologies represented.

If phylosymbiosis is observed, both host phylogenetic relatedness and ecology could be contributing to the pattern. Several studies have disentangled the effects of these two factors and have shown that phylosymbiosis can be observed among hosts that share habitats or diets, and among hosts that reside in different habitats and consume different diets. For example, in mice, voles, and shrews, gut microbiotas tend to be more similar among closely related host species, despite these animals occupying different habitats and consuming different diets [20]. In populations of American pikas (Ochotona princeps) from different mountain ranges, a cladogram of gut microbiota similarity was congruent with a phylogeny of host genetic similarity [21]. Among hosts with overlapping diets, gut microbiotas still exhibit patterns consistent with phylosymbiosis, as has been documented for folivorous primates [9]. Furthermore, in 33 species of sympatric herbivores from the Laikipia region in central Kenya, host phylogenetic relatedness strongly predicted gut microbiota composition $(r=0.91)$, which was weakly correlated with host diet $(r=0.28)$ [22], suggesting that convergence of gut microbiotas among closely related hosts was not primarily due to similarities in their diet.

Here, we build upon this work and use 16S rRNA gene sequencing to determine the relative influences of host phylogenetic relatedness and host ecology in structuring the gut microbiotas of 11 species of herbivores living sympatrically in the Masai Mara National Reserve (henceforth the Masai Mara) in southwestern Kenya. We survey the gut microbiotas of African buffalo, domestic cattle, common eland, impala, Kirk's dik-dik, Thompson's gazelle, topi, Masai giraffe, common warthog, plains zebra, and African elephant (Table 1). These species represent 5 mammalian families (Bovidae, Elephantidae, Equidae, Giraffidae, and Suidae) and three dietary guilds: grazers, browsers, and mixed-feeders. Furthermore, we compare the gut microbiotas of conspecific herbivores from the Masai Mara and Laikipia to determine the extent to which host geography and/or local habitat influence gut microbiota composition and patterns of phylosymbiosis. The two regions differ in their altitude, soils, rainfall, vegetation, mammal densities, and degree of human disturbance [23-27], any of which could potentially affect the gut microbiota compositions of their resident herbivores. Specifically, our study aims were to survey the gut microbiotas of 11 species of herbivores and 1) determine whether host phylogenetic relatedness or diet more strongly predict gut microbiota similarity among these hosts at broad taxonomic scales (i.e. among all study species) and lesser taxonomic scales (i.e. among 7 closely related Bovid species), 2) evaluate the influences of host taxonomy (family and species) and host dietary guild on gut microbiota composition and diversity, and 3) examine the amount of variance in the gut microbiota explained by host phylogeny and ecology (i.e., diet, geography) in conspecific hosts from the Masai Mara (this study) and Laikipia [22]. Collectively, our findings elucidate the factors shaping the gut microbiota of hosts at greater and lesser taxonomic scales.

\section{Results}

Aim 1: determine the strongest predictor of gut microbiota similarity among herbivore hosts at greater and lesser taxonomic scales

We conducted partial correlation coefficient tests to determine the relative contributions of host phylogenetic relatedness and diet in predicting gut microbiota structure. These tests evaluated the strength of the relationship between host phylogenetic relatedness and gut microbiota similarity (phylosymbiosis), while

Table 1 List of host study species and their associated metadata

\begin{tabular}{|c|c|c|c|c|c|}
\hline Order & Family & Species (common name) & Dietary Guild & Total Samples (N) & Analyzed samples (N) \\
\hline Cetartiodactyla & Bovidae & African buffalo & grazer & 18 & 17 \\
\hline Cetartiodactyla & Bovidae & Domestic cattle & grazer & 14 & 13 \\
\hline Cetartiodactyla & Bovidae & Common eland & mixed feeder & 8 & 8 \\
\hline Cetartiodactyla & Bovidae & Impala & mixed feeder & 20 & 20 \\
\hline Cetartiodactyla & Bovidae & Kirk's dik dik & browser & 37 & 31 \\
\hline Cetartiodactyla & Bovidae & Thomson's gazelle & mixed feeder & 14 & 14 \\
\hline Cetartiodactyla & Bovidae & Topi & grazer & 19 & 19 \\
\hline Cetartiodactyla & Giraffidae & Masai giraffe & browser & 25 & 18 \\
\hline Cetartiodactyla & Suidae & Warthog & mixed feeder & 9 & 8 \\
\hline Perissodactyla & Equidae & Plains zebra & grazer & 5 & 5 \\
\hline Proboscidea & Elephantidae & African elephant & mixed feeder & 12 & 12 \\
\hline
\end{tabular}


controlling for dietary similarity, and assessed the relationship between host dietary similarity and gut microbiota similarity, while controlling for phylogenetic relatedness. Phylogenetic relatedness was based on divergence times between host species and diet was quantified by \%C4 grass values in the diet (e.g. proportion of monocotyledon grasses consumed relative to trees and shrubs) previously published for these host species [2831] (Table S1).

Although a dendrogram of gut microbiota similarity did not closely reflect host phylogeny (Fig. 1a), partial correlation coefficient tests indicated that gut microbiota similarity increased with host phylogenetic relatedness even after controlling for dietary similarity (Table 2). Across the 11 herbivore species, the gut microbiotas were generally more similar among closely related host taxa (e.g., buffalo and cattle) than among distantly related host taxa (e.g., impala and elephant), and the average strength of the phylosymbiosis signal across microbiota similarity metrics was 0.74 (Fig. 1b). No relationship was observed between host dietary similarity (\%C4) and gut microbiota similarity at this broad taxonomic scale (Table 2). Importantly, at a lesser host taxonomic scale, among 7 closely related Bovid species, we observed the opposite patterns. Gut microbiota similarity did not covary with host phylogenetic relatedness after adjusting for dietary similarity among these bovid species (Fig. 1b), but we did find a significant relationship between dietary similarity and gut microbiota similarity (average $r=0.64$ ) (Table 2). In summary, across broad host taxonomic scales, host phylogenetic relatedness strongly predicted gut microbiota similarity, but host diet more strongly predicted gut microbiota structure at a lesser host taxonomic scale.

PERMANOVA analyses that included categorical variables for host taxonomy (family or species), dietary guild (grazer, browser, or mixed feeder) and sample month echoed the findings described above. Across the surveyed herbivores, host family explained on average $23 \%$ of the variation in gut microbiota structure, followed by host dietary guild (10\%), and sample month (8\%) (Table 3). Regardless of whether distance matrices took into account the presence/absence of bacterial taxa, their proportional abundances, or their phylogenetic relatedness, the percent variation explained by each host factor was consistent. Therefore, for brevity, we only present PCoA ordination plots using the Bray-Curtis index. These plots show that gut microbiotas primarily partition by host family, and also secondarily by host dietary guild (Fig. 1c). Additionally, we conducted the same PERMANOVA statistics from above but specified host species in lieu of the host family term; here, host species explained on average $30.92 \%$ of the variation across distance metrics, host diet explained $10.44 \%$ of the variation and sample month accounted for $7.93 \%$ of the variance (Table S2). Lastly, within Bovidae, host dietary guild was a slightly stronger predictor of the gut microbiota than host species or sample month. On average, host dietary guild accounted for $17.3 \%$ of the variation, whereas host species explained $12.2 \%$ of the variation, and sample month contributed to $7.3 \%$ of the variation (Table 3). PCoA ordinations showed that the gut microbiotas of bovids clustered by host dietary guild and host species (Fig. 1d). Collectively, our findings suggest that host phylogenetic relatedness and taxonomy predict gut microbiota structure across the studied herbivores, but among closely related host species, host diet is the more influential predictor.

Aim 2: evaluate the influences of host taxonomy and host dietary guild on gut microbiota composition and diversity Here, we compared gut microbiota taxonomic composition among the different hosts to identify the bacterial taxa that were characteristic of particular host families or dietary guilds. We also examine the extent to which host taxonomy (family or species) and dietary guild were associated with gut microbiota alpha-diversity.

\section{Microbiota composition}

Our analyses showed that some bacterial taxa were widely shared among host species and dietary guilds, whereas others were abundant only in particular host species. All herbivore gut microbiotas were dominated by two bacterial phyla, Firmicutes (51\% average relative abundance across samples), and Bacteroidetes (32\%) (Fig. $\mathrm{S} 1$ ). The most abundant bacterial families were Ruminococcaceae (30.8\%), Rikenellaceae (11.4\%), Lachnospiraceae (10.9\%), and Prevotellaceae (8\%) (Fig. 2a). Prevalent bacterial genera included Alistipes, Bacteroides, Ruminococcus, and Treponema (Fig. S2). Only 10 out of 11,930 (0.08\%) Amplicon Sequence Variants (ASVs) were present in $90 \%$ of samples pooled across all host species; 7 were assigned to the family Ruminococcaceae, 1 to Peptococcaceae, and 2 to Lachnospiraceae (Agathobacter). According to a BLAST search against the NCBI nucleotide database, sequences from the 7 Ruminococcaceae ASVs were highly similar to sequences from uncultured Ruminococcaceae strains, uncultured rumen bacteria, and uncultured anaerobic bacteria. Of these 10 ASVs, only two were abundant across samples (e.g., ASV10543 Ruminococcaceae), five were modestly abundant in specific host species (e.g., ASV71 Agathobacter in elephants), and 3 were present at very low abundances in all samples (e.g., ASV7824 Peptococcaceae) (Fig. S3). The latter 3 ASVs do not appear to represent contamination introduced during DNA extraction and sequencing, as these sequences are highly similar to those found in rumen and fecal samples. 


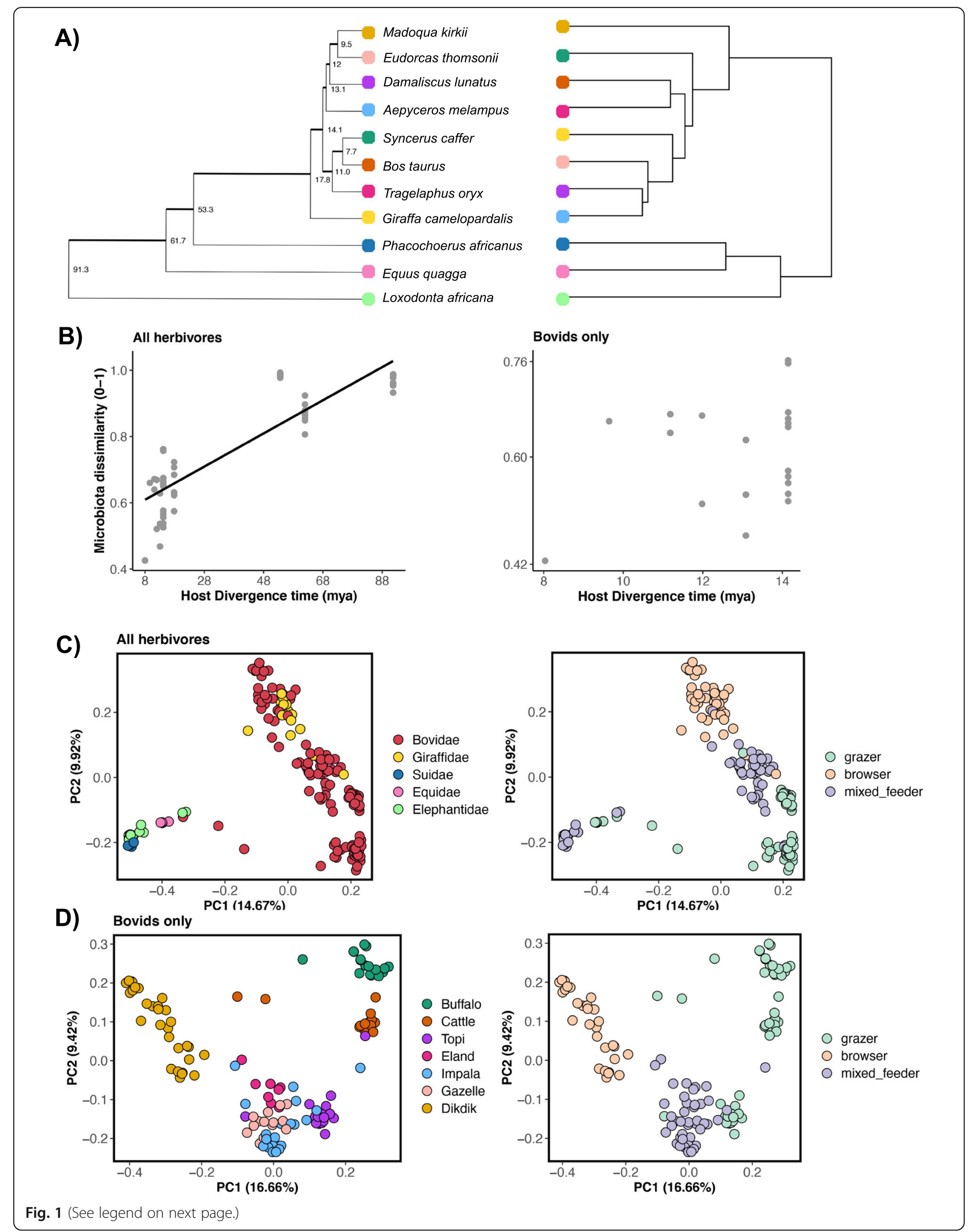


(See figure on previous page.)

Fig. 1 African herbivore gut microbiotas exhibit patterns consistent with phylosymbiosis. a Phylogenetic tree of host species (left) obtained from pruning Upham's et al. 2019 Mammalian supertree, compared against a dendogram (right) of gut microbiota similarity using hierarchical clustering. b Scatterplot of pairwise host divergence times (in millions of years) vs. gut microbiota similarity (Bray-Curtis distances) across all sampled herbivores (left) and within the single host family Bovidae (right). The plot on the left has a trendline representing the best fit line of a linear model regressing Bray-Curtis dissimilarity with host phylogenetic distance, which was added for plotting purposes. c PCoA plots constructed from Bray-Curtis dissimilarity matrices. Each point represents a sample and is color-coded by host family (left) or host dietary guild (right). Closeness of points indicates high community similarity. The percentage of variance accounted for by each principal-coordinate axis is shown in the axis labels. $\mathbf{d}$ PCoA plots constructed from Bray-Curtis dissimilarity matrices of bovid species only. Each point is color-coded by host species (left) or host dietary guild (right)

Nonetheless, variation in gut microbiota compositions among host families and dietary guilds was evident. Indicator species analysis showed that the gut microbiotas of elephants were significantly associated with Endomicrobiaceae and Desulfobulbaceae, those of zebras with Helicobacteraceae and Deltaproteobacteria, and those of warthogs with Myxococcales and Coxiellaceae (Fig. 2b). Giraffe gut microbiotas were highly associated with Enterobacteriaceae, Bifidobacteriaceae, and Bacillaceae. No bacterial taxa were strongly and specifically associated with Bovid hosts. Furthermore, there were bacterial types that were indicative of specific dietary guilds. Grazer gut microbiotas were characterized by Sphingobacteriaceae, Flavobacteriaceae, Neisseriaceae, and Lentisphaeria (Fig. 2c). Browser gut microbiotas had 11 indicator bacterial taxa, including Bacillaceae, Coriobacteriales, Methanomicrobia and Rubrobacteriaceae. Lastly, the gut microbiotas of mixed feeders were highly associated with Synergistaceae, Succinivibrionaceae, and Bacteroidales, among other bacteria.

More fine-scale analysis of the presence and absence of bacterial ASVs also revealed that the gut microbiotas of our studied herbivores contained microbes that were biased towards particular host species. These were bacterial ASVs that were present in $75 \%$ of samples for that host species, and absent in $97 \%$ of samples from other hosts. The gut microbiotas of buffalo, cattle, topi, and impala mostly contained ASVs that were present in other herbivores, as $<3 \%$ of their ASVs were biased towards any of these host species. Between 4 and $8 \%$ of ASVs comprising the gut microbiota of dik-diks, eland, elephant, Thompson's gazelle, and giraffe were biased towards these host species. Warthogs and zebras however, harbored more unusual microbiotas, as $70-77 \%$ of their ASVs were rarely detected in the guts of the other surveyed African mammals.

\section{Microbiota alpha-diversity}

Gut microbiota richness, evenness, and phylogenetic diversity also varied with host taxonomy (family and species) and dietary guild (Table 4, Fig. 3). Post-hoc comparisons revealed that hosts from the Suidae and Elephantidae families generally harbored less rich and less even gut microbiotas than the other surveyed host families (Fig. 3a; Table S3). Moreover, equids harbored more phylogenetically diverse (PD) gut communities than all other herbivores (Table S3). Across the three alpha-diversity metrics, browsers had less diverse gut microbiotas than grazers or mixed-feeders (Fig. 3a, Table S4). Similar tests that included host species in lieu of host family indicated that host species was a strong predictor of gut microbiota alpha-diversity (Chao1 $X^{2}=$ 134.42, Shannon diversity $X^{2}=45.86, \mathrm{PD} \chi^{2}=74.31$ ). Host dietary guild was also associated with microbiota

Table 2 The relative contributions of host phylogenetic relatedness and diet in predicting gut microbiota similarity

\begin{tabular}{|c|c|c|c|c|c|c|c|}
\hline & & \multicolumn{3}{|c|}{ Phylogenetic Relatedness } & \multicolumn{3}{|c|}{ Dietary Similarity (\% C4 grasses) } \\
\hline & & $\mathrm{R}$ & Z stat & p.val & $\mathrm{R}$ & Z stat & p.val \\
\hline \multirow[t]{4}{*}{ Across all host study species (11 sp.) $(N=165)$} & Bray-Curtis & 0.77 & 8.90 & $<0.0001$ & 0.15 & 1.09 & 0.27 \\
\hline & Jaccard & 0.72 & 7.50 & $<0.0001$ & 0.03 & 0.27 & 0.78 \\
\hline & Unifrac (weighted) & 0.76 & 8.51 & $<0.0001$ & 0.10 & 0.73 & 0.46 \\
\hline & Unifrac (unweighted) & 0.71 & 7.47 & $<0.0001$ & 0.02 & 0.152 & 0.87 \\
\hline \multirow[t]{4}{*}{ Across bovids (7 sp.) $(N=122)$} & Bray-Curtis & 0.43 & 2.07 & 0.05 & 0.68 & 3.98 & $<0.001$ \\
\hline & Jaccard & 0.35 & 1.63 & 0.12 & 0.69 & 4.05 & $<0.001$ \\
\hline & Unifrac (weighted) & 0.31 & 1.41 & 0.17 & 0.57 & 2.94 & $<0.001$ \\
\hline & Unifrac (unweighted) & 0.29 & 1.29 & 0.21 & 0.63 & 3.52 & 0.002 \\
\hline
\end{tabular}

Shown are the rho, test statistic, and $p$-values associated with partial correlation coefficient tests that evaluated the correlation between 2 variables (e.g. gut microbiome similarity and phylogenetic relatedness, while controlling for a third (e.g. dietary similarity). The tests were conducted on 4 types of gut microbiome distance metrics, and significant $p$-values are bolded 
Table 3 Host taxonomy and dietary guild shape the gut microbiotas of African herbivores

\begin{tabular}{|c|c|c|c|c|c|}
\hline Analysis & Host factors & $\begin{array}{l}\text { Bray-Curtis } \\
\text { (\% variance } \\
\text { explained) }\end{array}$ & $\begin{array}{l}\text { Jaccard } \\
\text { (\% variance } \\
\text { explained) }\end{array}$ & $\begin{array}{l}\text { Weighted Unifrac } \\
\text { (\% variance } \\
\text { explained) }\end{array}$ & $\begin{array}{l}\text { Unweighted } \\
\text { Unifrac } \\
\text { (\% variance } \\
\text { explained) }\end{array}$ \\
\hline \multirow[t]{3}{*}{$\begin{array}{l}\text { Across all host study species (11 sp.) } \\
(N=165)\end{array}$} & Host family & $\begin{array}{l}22.34 \\
p=0.001\end{array}$ & $\begin{array}{l}20.62 \\
p=0.001\end{array}$ & $\begin{array}{l}25.90 \\
p=0.001\end{array}$ & $\begin{array}{l}24.29 \\
p=0.001\end{array}$ \\
\hline & $\begin{array}{l}\text { Host dietary } \\
\text { guild }\end{array}$ & $\begin{array}{l}11.20 \\
\boldsymbol{p}=\mathbf{0 . 0 0 1}\end{array}$ & $\begin{array}{l}10.17 \\
p=\mathbf{0 . 0 0 1}\end{array}$ & $\begin{array}{l}10.04 \\
p=0.001\end{array}$ & $\begin{array}{l}10.30 \\
p=0.001\end{array}$ \\
\hline & sample month & $\begin{array}{l}7.39 \\
p=0.001\end{array}$ & $\begin{array}{l}6.78 \\
p=0.001\end{array}$ & $\begin{array}{l}9.90 \\
p=0.001\end{array}$ & $\begin{array}{l}7.68 \\
p=0.001\end{array}$ \\
\hline \multirow[t]{3}{*}{ Across bovids (7 sp.) $(N=122)$} & $\begin{array}{l}\text { Host dietary } \\
\text { guild }\end{array}$ & $\begin{array}{l}18.26 \\
p=0.001\end{array}$ & $\begin{array}{l}15.91 \\
p=0.001\end{array}$ & $\begin{array}{l}18.35 \\
p=0.001\end{array}$ & $\begin{array}{l}16.77 \\
p=0.001\end{array}$ \\
\hline & Host species & $\begin{array}{l}15.16 \\
p=0.001\end{array}$ & $\begin{array}{l}13.38 \\
p=0.001\end{array}$ & $\begin{array}{l}8.23 \\
p=0.001\end{array}$ & $\begin{array}{l}12.16 \\
p=0.001\end{array}$ \\
\hline & sample month & $\begin{array}{l}7.56 \\
p=0.001\end{array}$ & $\begin{array}{l}7.03 \\
p=0.001\end{array}$ & $\begin{array}{l}7.59 \\
p=0.001\end{array}$ & $\begin{array}{l}7.17 \\
p=0.001\end{array}$ \\
\hline
\end{tabular}

Shown are the $\mathrm{R}^{2}$ values (\% variance explained) and $p$-values for PERMANOVA tests ( $\mathrm{y} \sim$ sample month + host dietary guild + host taxonomy) based on 4 types of distance matrices. Bray-Curtis and Weighted Unifrac distance matrices take into consideration the proportions of bacterial taxa, while Jaccard and unweighted Unifrac take into account only their presence or absence. Both Unifrac distances account for phylogenetic relatedness among bacterial types. Significant $p$-values $(a=0.05)$ are bolded

alpha-diversity, but with a lower effect size (Chao1 $\chi^{2}=$ 16.86, Shannon diversity $\mathrm{X}^{2}=14.26$, PD $\mathrm{X}^{2}=5.85$; all $p<$ 0.001). Post-hoc comparisons indicated that warthogs, giraffes, and elephants harbored less rich and phylogenetically diverse gut microbiotas than most bovids, whereas zebras harbored the richest gut microbiotas of the host species surveyed (Table S5). Not as many species-specific differences were observed when examining microbiota evenness (Table S5).

Within a single host family (i.e. Bovidae), the gut microbiotas of buffalo, dikdiks, and gazelles were less rich, even, and phylogenetically diverse than those of all other sampled bovids (Fig. 3b, Table S6). In these bovid hosts, browsers also had less diverse gut microbiotas than grazers or mixed-feeders (Fig. 3b, Table S4). Additionally, we determined whether similarity in gut microbiota alpha-diversity was correlated with dietary similarity (\%C4 grasses in diet), while accounting for variation in the gut microbiota associated with host phylogenetic relatedness. Gut microbiota evenness (Shannon diversity) increased with host dietary similarity, but this was not true of gut microbiota richness or phylogenetic diversity (partial Mantel Chao1 $r=0.03$, $p=0.34$; Shannon $r=0.35, p=0.028$, PD $r=0.097, p=$ $0.25)$.

\section{Aim 3: examine the amount of variance in the gut microbiota explained by geographic region among conspecific hosts}

Lastly, we further analyzed the influences of host phylogenetic relatedness and host ecology (i.e., diet and geography) on the gut microbiota of 8 herbivore species from two distinct populations in the Masai Mara (this study) and Laikipia (Kartzinel et al. [22]). The eight herbivore species overlapping both studies were African buffalo, domestic cattle, common eland, impala, giraffe, plains zebra, common warthog, and African elephant. Four of these 8 species were bovids (buffalo, cattle, eland, and impala).

We found that gut microbiota structure differed little between conspecific hosts from the two geographic regions of Kenya, as this factor accounted for $<3 \%$ of the variation in the gut microbiota, on average (PERM ANOVA analyses, Table S7). The gut microbiotas were primarily structured by host species and host dietary guild, which explained on average, 38 and $11 \%$ of the variation, respectively (PERMANOVA analyses, Table S7); sample month explained an additional 7\% of the variation. Ordination plots confirm these findings and demonstrate that samples primarily cluster by host species (Fig. 4a), although some separation of samples based on host geographic region is also observed, particularly among cattle, impala, and giraffe. Patterns consistent with phylosymbiosis were also observed in this combined dataset, despite herbivore hosts occupying habitats in Kenya separated by over $300 \mathrm{~km}$, and representing distinct populations. Gut microbiota similarity increased with host phylogenetic relatedness even after accounting for variation attributable to host diet (\%C4 grasses) (Table S8), although the strength of the phylosymbiosis signal $(r=0.62)$ was less than that obtained for Masai Mara herbivores only $(r=0.74)$, or the value that was previously reported for the Laikipia herbivores $(r=0.91)$ [22]. No relationship was observed between gut microbiota similarity and dietary similarity after controlling for variation due to host phylogeny. Among the four species of bovids that overlapped between the two studies, neither host phylogenetic relatedness nor 


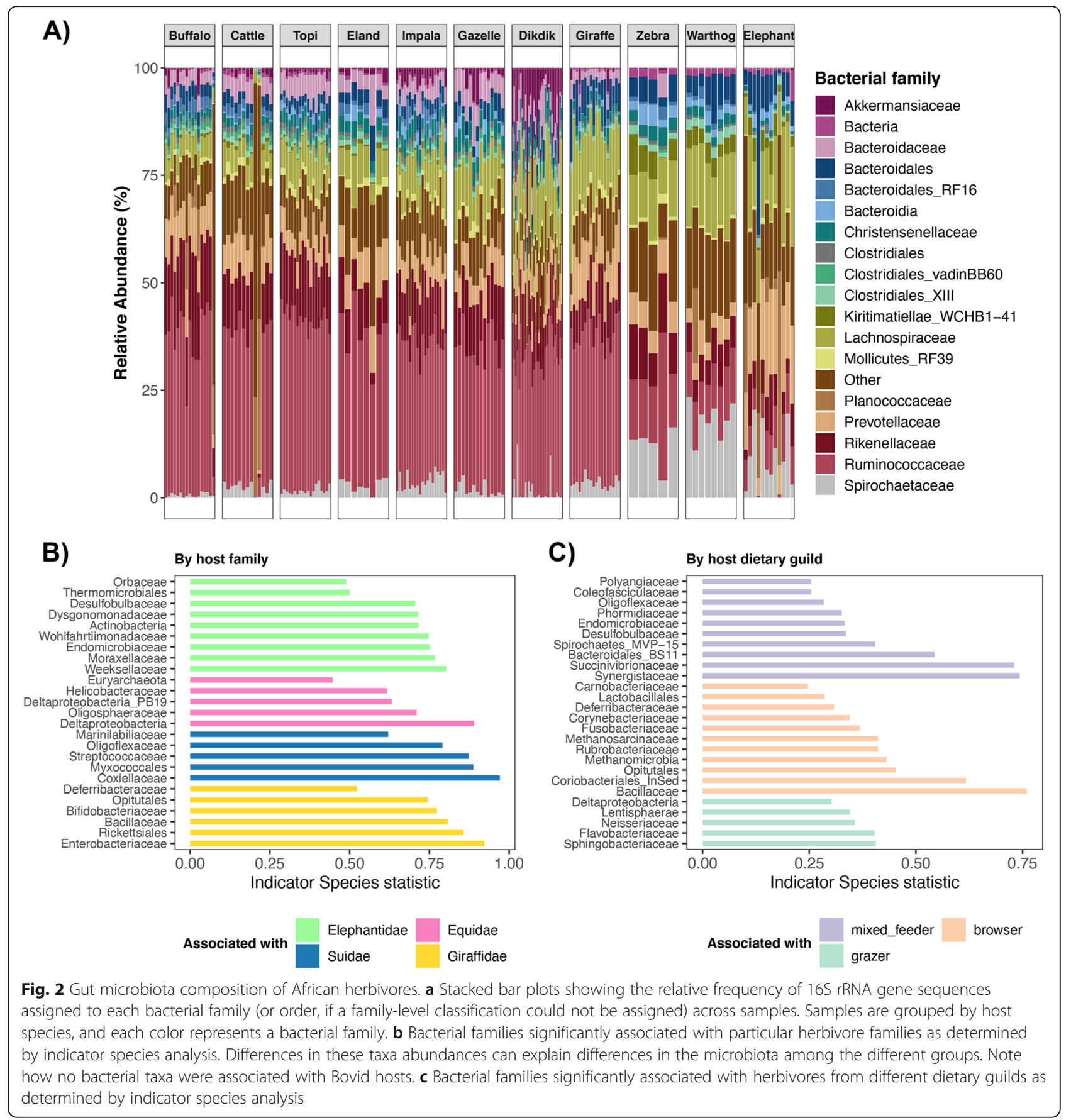

host diet (\%C4) significantly predicted gut microbiota similarity (Table S8). The latter findings should be interpreted with caution, as only 4 host species were sampled, whereas the same analyses had a larger sample size ( 7 host species) when conducted solely on our Masai Mara dataset.

In this combined dataset, only 3 of $18,039(0.01 \%)$ ASVs were present across $90 \%$ of samples; two were classified as Ruminococcaceae, and 1 as Lachnospiraceae. All 3 ASVs were among the 10 ASVs also present across $90 \%$ of Masai Mara samples. To further compare the gut microbiotas of conspecific hosts, we visualized the relative abundances of the 32 most abundant ASVs in the dataset. A heatmap of these 32 ASVs demonstrate that there were ASVs that were found in comparable proportions in conspecific hosts, as well as ASVs that were differentially abundant among conspecific hosts (Fig. 4b). For example, ASV4033 Prevotellaceae is similarly abundant between buffalo, cattle, and giraffe in the Masai Mara and 
Table 4 Microbiota richness, evenness, and phylogenetic diversity vary with host taxonomy and dietary guild

\begin{tabular}{|c|c|c|c|c|}
\hline Model & Factor & Chao 1 Richness & Shannon diversity & Phylogenetic diversity \\
\hline \multirow[t]{2}{*}{$\begin{array}{l}\text { Across all study sp. } \\
\text { (11 sp.) }(N=161)\end{array}$} & Host family & $\begin{array}{l}X^{2}=53.58 \\
\boldsymbol{p}<0.001\end{array}$ & $\begin{array}{l}x^{2}=33.45 \\
p<0.001\end{array}$ & $\begin{array}{l}X^{2}=18.31 \\
\boldsymbol{p}=\mathbf{0 . 0 0 1}\end{array}$ \\
\hline & Host dietary guild & $\begin{array}{l}x^{2}=79.03 \\
\boldsymbol{p}<0.001\end{array}$ & $\begin{array}{l}x^{2}=73.72 \\
\boldsymbol{p}<0.001\end{array}$ & $\begin{array}{l}x^{2}=52.61 \\
p<0.001\end{array}$ \\
\hline \multirow[t]{2}{*}{$\begin{array}{l}\text { Within bovids } \\
\text { (7 sp.) }(N=118)\end{array}$} & Host species & $\begin{array}{l}X^{2}=57.03 \\
\boldsymbol{p}<\mathbf{0 . 0 0 1}\end{array}$ & $\begin{array}{l}X^{2}=10.81 \\
\boldsymbol{p}=\mathbf{0 . 0 2}\end{array}$ & $\begin{array}{l}X^{2}=50.53 \\
\boldsymbol{p}<\mathbf{0 . 0 0 1}\end{array}$ \\
\hline & Host dietary guild & $\begin{array}{l}X^{2}=19.55 \\
\boldsymbol{p}<0.0001\end{array}$ & $\begin{array}{l}x^{2}=33.91 \\
p<0.0001\end{array}$ & $\begin{array}{l}X^{2}=10.67 \\
\boldsymbol{p}<0.01\end{array}$ \\
\hline
\end{tabular}

Shown are the likelihood ratio $x^{2}$ test statistics obtained for linear mixed effects models specifying host dietary guild and host family as predictor variables, sample date as a random effect, and an alpha-diversity metric as a dependent variable. A similar model restricted to bovids was also constructed; it specified host species instead of host family. Significant $p$-values $(\alpha=0.05)$ are bolded

\section{A) All hervibores}
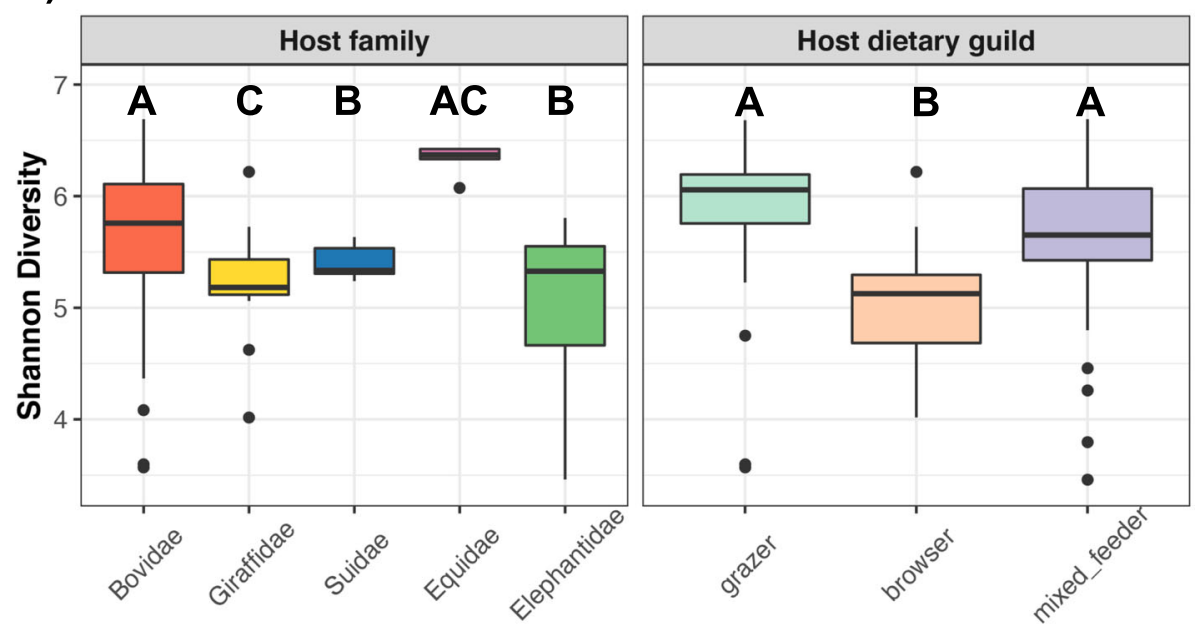

B) Bovids only
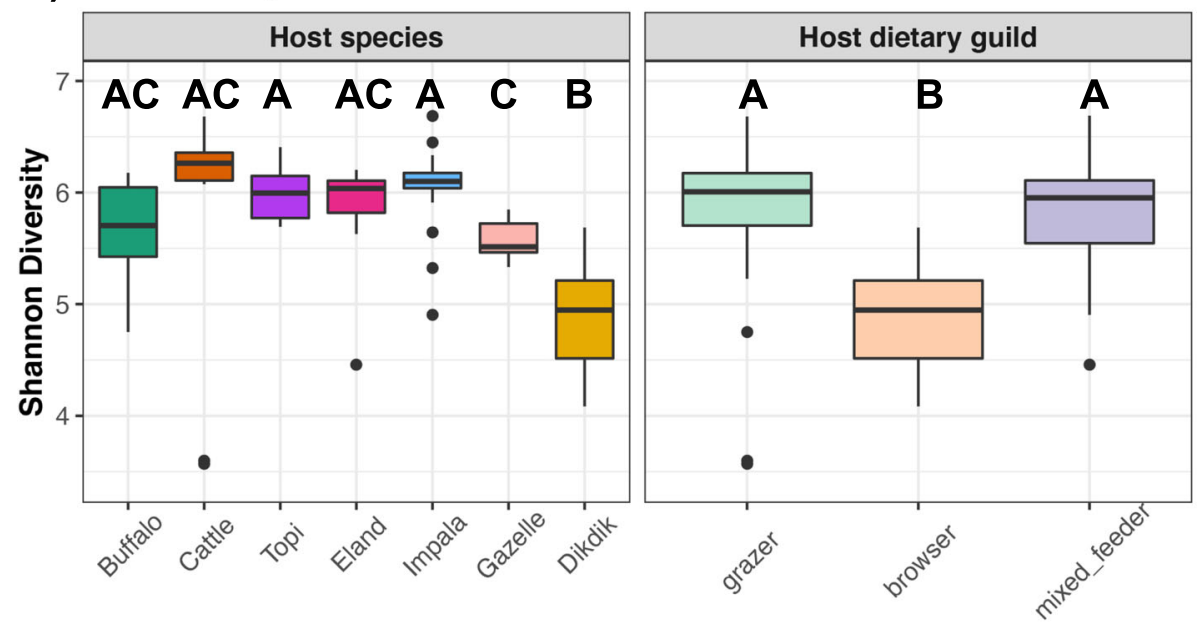

Fig. 3 Host taxonomy and dietary guild are associated with gut microbiota diversity in African herbivores. a Boxplots of microbiota evenness (Shannon diversity) among host families and dietary guilds across all studied herbivores, and $\mathbf{b}$ ) among host species and dietary guilds within the family Bovidae. Boxes that do not share any letters represent statistically significant comparisons; see Tables S3-S6 for all post-hoc comparisons.

Thicker dots represent outlier values 


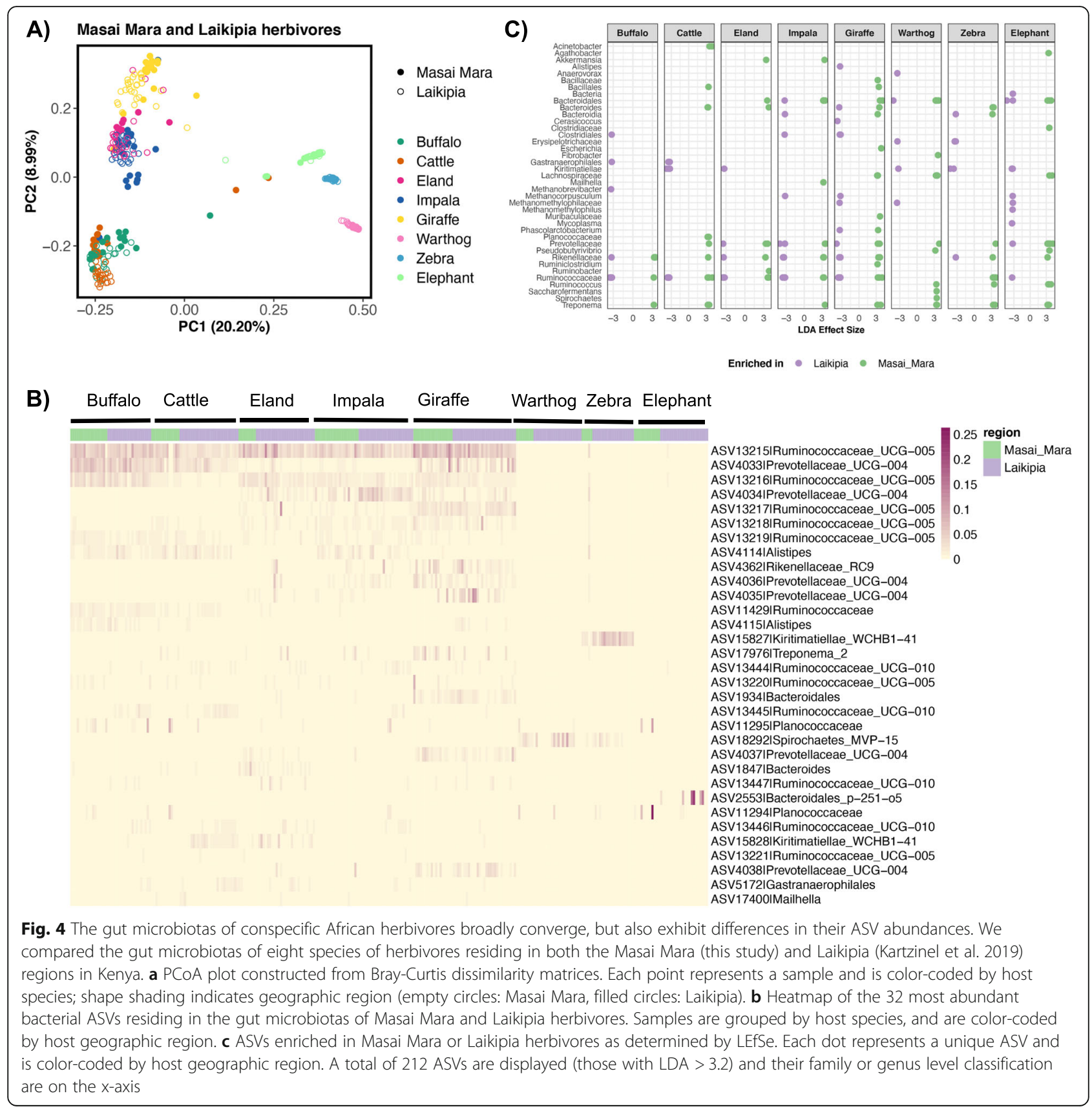

Laikipia, but ASV15828 Kiritimatiellae appears to be enriched in cattle from Laikipia compared to cattle from the Masai Mara.

To further extend these analyses and identify additional ASVs that may be differentially abundant among conspecifics (e.g., Masai Mara elephants vs. Laikipia elephants), we also conducted Linear Discriminant Analysis Effect Size (LEfSe) for each host species. Roughly $30 \%$ of the ASVs in the gut microbiotas of each host species were enriched in hosts from one population relative to the other (Table S9). ASVs that were typically enriched were classified as Ruminococcaceae, Lachnospiraceae,
Rikenellaceae, Clostridiales, Bacteroidales, and Kiritimatiellae (Fig. 4c). Herbivore species from Laikipia tended to be enriched in Methanocorpusculum, Clostridiales, and Kiritimatiellae ASVs relative to Masai Mara herbivores (Fig. 4c). Masai Mara gut microbiotas were overrepresented by Lachnospiraceae and Treponema ASVs. Interestingly, hosts from both geographic regions could be enriched in taxonomically similar ASVs. For example, eland in Laikipia were enriched in 3 ASVs classified as Prevotellaceae, Rikenellaceae, and Ruminococcaceae, respectively, and eland from the Masai Mara were enriched in 3 different ASVs that were also classified as 
Prevotellaceae, Rikenellaceae, and Ruminococcaceae (Fig. 4c). These findings suggest that variation in the gut microbiotas of these herbivore conspecifics is observable at the level of specificity of bacterial ASVs.

\section{Discussion}

\section{Principal findings of study}

The primary purpose of this study was to determine the relative contributions of host phylogenetic relatedness and dietary guild in structuring the gut microbiotas of 11 species of sympatric African herbivores. We also compared the gut microbiotas of herbivores from the Masai Mara to herbivores from Laikipia, Kenya to determine the extent to which two distinct populations of identical herbivore species varied in their gut microbiotas. We found that gut microbiotas were highly speciesspecific, but also varied with host ecology, including host diet and sample month, particularly among closely related Bovid species. Furthermore, gut microbiota similarity increased with host phylogenetic relatedness at a relatively broad host taxonomic scale, but at a lesser taxonomic scale, host diet (\%C4 grasses) was the strongest predictor of gut microbiota similarity. Lastly, although the gut microbiotas of conspecific herbivore hosts converged and primarily clustered by host species, variation among conspecifics in the relative abundances of their bacterial ASVs were also observed. Collectively, our findings suggest that mammalian gut microbiotas are strongly shaped by host phylogenetic relatedness and taxonomy, but they can be further modified by host ecology, including host diet and geography.

\section{Aim 1: determine the strongest predictor of gut microbiota similarity among herbivore hosts at greater and lesser taxonomic scales}

Our results showed that phylosymbiosis was observed across the relatively broad host taxonomic scale encompassing multiple herbivore families, i.e. among 11 species of herbivores living sympatrically in the Masai Mara. Patterns of phylosymbiosis have been documented extensively in many vertebrate groups, including primates, rodents, ruminants, carnivores, reptiles, and insects [912, 22, 32, 33]. Evidence of phylosymbiosis among host species living in sympatry specifically, has been previously documented in seven species of deer mice [34], six species of Malagasy mammals [35], twelve species of lemurs [36], and nine species of diurnal, non-human primates [37].

The mechanisms and processes that yield patterns of phylosymbiosis have not yet been elucidated, but host ecological and phenotypic traits are likely acting as filters and thus shaping microbial community assembly. Closely related hosts are potentially colonized by taxonomically similar microbes due to similarities in their morphology, anatomy, digestive physiologies, and immune system components [38-40]. Specifically, related hosts may possess similar antimicrobial peptides and toll-like receptors that serve to filter the same bacterial clades from the environment [41, 42]. Closely related hosts may further develop immune tolerance via adaptive immunity to the same symbiotic, commensal, and transient microbes [41, 42]. Lastly, some closely related hosts may also possess similar social group structures and pathways for transmitting microbes among groupmates, thereby contributing to patterns of phylosymbiosis [43-46]. Overall, accumulation of differences in traits as hosts diverged from one another could potentially provide enough niche differentiation in the gut to promote the divergence of their symbiotic bacterial communities.

At a lower host taxonomic scale, within our sampled group of closely related Bovid species in the Masai Mara, variation in the gut microbiota was more strongly associated with host diet than host species, and we did not detect a pattern congruent with phylosymbiosis with this dataset. Similarly, other studies report that among closely related hosts, host ecology more strongly predicts the structure of the gut microbiota than host relatedness. For example, in lemurs (Eulemur spp., Propithecus spp.), phylosymbiosis was observed across but not within two host lineages, and within host lineages, host habitat (dry forest vs. rainforest) was significantly correlated with gut microbiota diversity [36]. In populations of yellow (Papio cynecephalus) and anubis baboons (Papio anubis), gut microbiota dissimilarity did not increase with host genetic distance, but did vary with their habitat's soil chemistry [47]. Because the bovids surveyed here are closely related, their gut microbiotas are already very similar, and variation can result from fine-scale differences in diet (proportions of grass vs. shrubs vs. trees consumed) [48-51]. Nonetheless, some of the variation in the gut microbiota of bovids is not attributable to host diet and can be explained by host phylogenetic relatedness. Thus, even among closely related host species, both ecological and evolutionary forces shape gut microbial communities.

Aim 2: evaluate the influences of host taxonomy and host dietary guild on gut microbiota composition and diversity Across the surveyed herbivores, gut microbiota composition, diversity, and structure varied with host taxonomy. Species-specificity of the gut microbiota is widespread, and is commonly reported in the majority of comparative gut microbiome studies [11, 52-54]. Host species may vary in their body size, behavior, neuroendocrine system, immune function, and metabolism, any of which could potentially influence the structure of their gut microbiotas $[39,40,55,56]$. When comparing gut 
microbiota alpha-diversity, results showed that warthogs and elephants harbored less diverse gut communities than did the other sampled herbivores. Due to their omnivory, warthogs have a greater dietary breadth than the other studied herbivores, yet they harbored less diverse microbiotas. This is in accordance with prior findings, which report that the most diverse diets do not always correlate with the most diverse gut microbiotas $[22,57,58]$. Furthermore, analyses showed that browsers had the least diverse gut microbiotas, potentially because they consume vegetation that has a higher lignin content and a lower fiber digestibility than grass [50]. Specialized bacterial metabolisms may be required to digest this tougher plant material. Additionally, group size has been shown to correlate with gut microbiota diversity $[59,60]$, and the browsers in our study (giraffes, dikdiks) typically forage in smaller groups than do grazers (buffalo, zebras) and mixedfeeders (gazelles, impala), which forage in herds. Frequent social interactions and interactions with a greater number of individuals is known to promote species richness in individual gut microbiotas [60, 61].

Similar to findings from a plethora of microbiome studies, the gut microbiota structure of the studied herbivores also varied with host diet. Hosts from each dietary guild consume food sources that vary in their structure, chemistry, and nutrition quality; these require morphological, physiological and behavioral adaptations $[62,63]$. For example, grazers mostly feed on grasses, which have thicker cell walls, a lower protein content, and use the $\mathrm{C}_{4}$ photosynthetic pathway compared to the leaves, shrubs, and woody vegetation consumed by browsers, which have a higher protein content, and use $C_{3}$ photosynthesis [62, 63]. To efficiently extract energy from these different food sources, browsers and grazers evolved adaptations in their salivary chemistry, tooth morphology, gut structure, and speed of digestion [64, 65]. These adaptations, along with the actual nutrients hosts are providing to their microbes, potentially contribute to gut microbiota divergence among hosts from different dietary guilds.

Despite differences in the gut microbiota among host species and dietary guilds, there were some features of the gut microbiota that were shared across individuals from multiple species. Across our surveyed herbivores, the most abundant bacterial taxa in the gut microbiota were Ruminococcaceae, Rikenellaceae, Lachnospiraceae, and Prevotellaceae which represent core taxa previously found in the gut microbiotas of many ruminants and herbivores in general, including cervids and bovids [8, 66], equids [67], elephants [68], and giraffes [69]. Ruminococcaceae and Lachnospiraceae have also been found in the guts of folivorous primates [3] and in domestic pigs [70, 71]. Members of these bacterial families are responsible for digesting the cellulose, hemicellulose, lignin, and protein found in vegetation, and fermenting these into short-chain fatty acids (SCFAs) [72]. SCFAs represent usable forms of energy for the hosts [73] and contribute to host colonocyte growth, communication, immune defense, and anti-inflammatory responses [1]. These bacterial taxa also possess fiber-degrading capabilities and can provide their hosts with protection against ingested toxic plant secondary metabolites [74]. Interestingly, 7 of the 10 ASVs that were present in $90 \%$ of Masai Mara herbivores were classified as Ruminococcaceae and were sequences highly similar to uncultured Ruminococcaceae strains extracted from bovine, ovine, and caprine rumens [75], suggesting that these "core" microbes may be functionally important for the host, and/or are easily acquired from the environment.

\section{Aim 3: examine the amount of variance in the gut microbiota explained by geographic region among conspecific hosts}

While gut microbiota structure was primarily associated with host species and phylogeny in the combined Masai Mara and Laikipia dataset, differences in gut microbiota composition between conspecific hosts from the two populations were also evident. Herbivores of the same species may possess similar evolutionary trajectories, physiologies, and behaviors, and thus may be providing microbes with similar niches for colonization, which is why at a broad level their gut microbiotas converge. However, the two geographic regions do vary in their climate, soil geochemistry, plant communities, and resident herbivore species [23-27, 76], and potentially in their bacterial species pools, which could lead to the fine-scale microbiota differences among conspecifics. This finding was supported by our data; according to LEfSe analyses, over $30 \%$ of ASVs were differentially enriched between Masai Mara and Laikipia hosts. Laikipia herbivores for example, tended to be enriched in ASVs classified as Methanocorpusculum, Clostridiales, and Kiritimatiellae, while Masai Mara herbivores had an overrepresentation of ASVs belonging to Lachnospiraceae and Treponema. Abundances of the methanogenic Methanocorpusculum are related to forage type and geographic location in cattle [77], and in the mammalian gut, Lachnospiraceae are associated with a high-fat diet [78]. Furthermore, in the bovine rumen, Treponema degrade hemicellulose and their growth increases in the presence of pectin $[79,80]$, a carbohydrate abundant in non-woody plants. This suggests that differences in the ASV abundances between conspecific hosts likely reflect fine-scale differences in their diets and habitats. Interestingly, both Clostridiales and Lachnospiraceae are major microbial taxa of the mammalian gut and comprise fermentative bacteria that synthesize SCFAs from the hydrolysis of starches and sugars [72]; thus, conspecific 
hosts can be enriched in taxonomically distinct microbes that perform similar functions. Future studies should examine whether phylosymbiosis is evident at the functional level in the gut metagenomes of African herbivores and in metazoan taxa in general. A recent study by Milani and colleagues reports that gut microbiome functional profiles varied with host dietary category (carnivore, piscivore, herbivore) across 24 species of mammals [81]. These gut microbiome functional profiles might also vary with host phylogeny. Such studies will be necessary to further our understanding of the processes and mechanisms potentially underlying patterns of phylosymbiosis.

Lastly, we found that phylosymbiosis was also evident among conspecific African herbivores living in allopatry, although the strength of the phylosymbiotic signal was slightly reduced compared to that observed for either sympatric population considered in isolation. Overlap in gut microbiota structure is thought to be lower in allopatric animal populations than in sympatric animals due to variation introduced by habitat, dietary differences, and the spatial limits of bacterial dispersal [12]. It is important to note that differences between the gut microbiotas of Laikipia and Masai Mara conspecifics could also be potentially attributable to differences in sampling, DNA extraction, and sequencing protocols between the two studies [82-84]. Collectively, our findings show that mammalian gut microbiotas converge among closely related host species and among conspecifics, but can be differentiated with variation introduced by the host's ecology.

\section{Conclusions}

Our study showed that among 11 species of African herbivores living in sympatry, gut microbiotas are highly species-specific and exhibit patterns congruent with phylosymbiosis. However, these gut microbiotas are also shaped by their host's ecology, and within closely related bovid host species, gut microbiota similarity is strongly predicted by host diet (\%C4 grasses in diet and dietary guild) and is not associated with host phylogenetic relatedness. Furthermore, among eight species of herbivores residing in two geographic regions in Kenya, gut microbiotas were similar among hosts of the same species, but also exhibited fine scale differences in the abundances of their bacterial ASVs. Overall, these findings suggest that related hosts are providing microbes with similar niches for colonization, but these microbial niches are further shaped by host diet, geography, and local environmental conditions.

\section{Methods}

\section{Study location and sampling}

Fecal samples $(N=181)$ were collected opportunistically from 11 species of herbivores permanently residing in the Talek and Mara Triangle regions of the Masai Mara $\left(1^{\circ} 22^{\prime} 19^{\prime \prime} \mathrm{S}, \quad 34^{\circ} \quad 56^{\prime} 17^{\prime \prime} \mathrm{E}\right)$ from March-June 2018 (Table 1). This Reserve is covered by open rolling grassland interspersed with seasonal watercourses and riparian vegetation. It has two rainy seasons (March-May and November-December, with annual rainfall $>1000 \mathrm{~mm}$ ) [85], and $81 \%$ of our sampling took place during the rainy months, particularly during the month of March (Fig. S4). Although the Masai Mara is home to small resident populations of zebra and wildebeest, millions of these individuals migrate into the Reserve from July-October every year. Because our sampling occurred before July, samples from wildebeest and zebras were limited.

For fecal sample collection, we either observed animals defecating or identified species-of-origin based on the size, shape, and consistency of fresh dung, following Kartzinel et al. [22]. Samples were then placed in sterile cryogenic vials and stored in liquid nitrogen until they were transported on dry ice to Michigan State University, where they remained frozen at $-80{ }^{\circ} \mathrm{C}$ until nucleic acid extraction. For a list of samples and their associated metadata, see the Github repository for this project (https://github.com/rojascon/Rojas_et_al_2020_African_ herbivores_gut_microbiome).

While we did not directly collect diet data from the surveyed herbivores, we used Kingdon's East African Mammals [86-89] to classify our study species into grazers, browsers, and mixed-feeders. To obtain more fine-scale data on host diet, we also compiled dietary C4 (\%) data for these herbivores from previously published studies (Table S1) [28-31]. Percent C4 values reflect the proportion of monocotyledon grasses consumed relative to trees, shrubs, and forbs.

\section{DNA extraction and 16S rRNA gene sequencing}

Fecal samples were sent to the University of Illinois at Chicago (UIC) Sequencing Core for automated DNA extractions using QIAGEN DNeasy PowerSoil kits (Valencia, CA, USA). DNA concentrations of the fecal sample extracts were quantified using Qubit. The V4 region of the $16 \mathrm{~S}$ rRNA gene was targeted for sequencing on the Illumina MiSeq platform at the Michigan State University Genomics Core, using published protocols by Caporaso et al. 2012 [90] and Kozich et al. 2013 [91].

\section{Sequence processing and bioinformatics}

Sequences from Masai Mara herbivore gut microbiotas were processed in $\mathrm{R}$ (v.3.6.2) [92] using the Divisive Amplicon Denoising Algorithm (DADA2) pipeline (v.1.14.1) [93] to infer amplicon sequence variants (ASVs). Briefly, reads were filtered for quality, allowing for 2 and 3 errors per forward and reverse read, respectively $($ trimLeft $=c(10,10), \max N=0, \operatorname{maxEE}=2, \operatorname{trunc} \mathrm{Q}=2)$. 
Forward reads were trimmed to $240 \mathrm{bp}$ and reverse reads to $200 \mathrm{bp}$; these paired-end reads were merged. Sequences were then dereplicated to remove redundancy and ASVs were inferred by pooling reads from all samples. Prior to creating the ASV abundance table, chimeras were removed and ASVs were taxonomically classified using the SILVA rRNA gene reference database (v.132) [94] with an $80 \%$ confidence threshold. ASVs taxonomically assigned as Eukarya, Chloroplasts, or Mitochondria were removed from the dataset, as were those of unknown Kingdom origin; 12,938 total ASVs remained. The resulting ASV table and the taxonomic designations of the ASVs are available on GitHub. On average, samples retained over $70 \%( \pm 11 \%)$ of their total sequences after processing in DADA2. Nineteen samples did not amplify well $(<400$ sequences after processing) and were removed from the dataset. Most of these samples belonged to browser species (giraffes and dik-diks), suggesting that there may have been PCR inhibitors in their fecal samples (e.g., humic acid, tannins) that prevented successful extraction of DNA or library preparation. Table 1 has the sample sizes $(\mathrm{N})$ for each study species before and after this filtering.

\section{Microbiota composition analyses}

Statistical analyses and data visualization were completed in $R$ unless otherwise stated. To visualize microbiota composition, stacked barplots were constructed in ggplot2 (v.3.3.2) [95]. These plots showed the bacterial phyla, families, and genera with average relative abundances greater than $1 \%$ across samples. We also identified the ASVs $(N=10)$ that were present in $>90 \%$ of samples across all host species, and the relative abundances of these ASVs were displayed as heatmaps using the $R$ pheatmap package (v.1.0.12) [96]. Sequences from the 10 ASVs were BLASTed against the National Center for Biotechnology Information (NCBI) Nucleotide database [75] to find similar biological sequences from known bacterial taxa. Furthermore, we also identified ASVs that were biased towards particular host species; these were ASVs that were present in $>75 \%$ of the samples for a particular host species (e.g., giraffes) and absent in $97 \%$ of samples from the other host species.

To detect the bacterial taxa strongly associated with particular host families or dietary guilds, we used the $R$ indicspecies package (v.1.7.9) [97], which calculates an indicator value for each bacterial taxon based on its prevalence in a given group and absence in others. A table of bacterial family relative abundances was used as input, and significance was assessed via permutation tests using 999 permutations $(\alpha=0.05)$. Bacterial families with indicator values $>0.4$ were plotted in ggplot2.

\section{Microbiota a-diversity statistical analyses}

Prior to alpha-diversity analyses, we controlled for the potential influences of sequencing depth by subsampling all samples to 17,000 sequences using the mothur (v.1.42.3) [98] sub.sample command. Four fecal samples did not meet this sequence cutoff criterion and were excluded from all alpha-diversity analyses. Mothur was used to construct rarefaction curves of ASV richness vs. sequencing depth (Fig. S5) and Good's coverage values averaged $97.78 \pm 0.91$ across all samples, indicating that sample coverage was high and appropriate for characterizing fecal microbiota profiles. These values are comparable to those typically reported in other mammalian gut microbiota studies $[8,99,100]$.

Microbiota alpha-diversity was estimated using Chao1 Richness, Shannon diversity, and Faith's Phylogenetic Diversity (PD) in R. Chao1 Richness and Shannon indices were calculated using the phyloseq package (v.1.33.0) [101]. To obtain measures of Faith's PD, we constructed a phylogenetic tree of ASV sequences using phangorn (v. 2.5.5) [102] and calculated PD using the picante package (v.1.8.1) [103]. The effects of predictor variables on each measure of alpha-diversity across all samples were evaluated via linear mixed models (LMMs) using the lme4 package (v.1.1.23) [104], specifying host dietary guild and host family as fixed variables and sample month as a random effect. A similar model that included host species as a predictor in lieu of host family was also evaluated. A third model was built for bovid samples only, which included host species and dietary guild as predictors. The significance of each predictor variable was determined by calculating likelihood ratio $X^{2}$ test statistics $(\alpha=0.05)$ on the full models using the car package (v.3.0.7) [105]. These tests were followed by TukeyHSD post-hoc tests with Benjamini-Hochberg adjustments to control for multiple comparisons. Boxplots of microbiota alpha-diversity were generated in ggplot2.

To further quantify the influence of host diet on the three metrics of gut microbiota alpha-diversity, we conducted partial Mantel tests with 999 permutations using the $R$ vegan package (v.2.5.7) [106]. Specifically, we evaluated whether similarity in gut microbiota alphadiversity was associated with similarity in dietary $\mathrm{C} 4(\%)$ after accounting for variation due to host phylogenetic relatedness. The 3 matrices used as input were i) a dissimilarity matrix of gut microbiota alpha-diversity, ii) a dissimilarity matrix of host \% $\mathrm{C} 4$, and iii) a matrix of host divergence times.

\section{Microbiota $\beta$-diversity analyses and testing for phylosymbiosis}

In order to determine the relative contributions and amount of variance explained by host predictor 
variables, permutational multivariate analyses of variance (PERMANOVA) tests based on Bray-Curtis, Jaccard, and Unifrac distance matrices were run using vegan. Bray-Curtis/Jaccard distances were estimated using vegan, whereas weighted and unweighted Unifrac distances were estimated using phyloseq. Bray-Curtis and weighted Unifrac distances take into account the abundances of bacterial taxa while Jaccard and unweighted Unifrac metrics only consider their presence or absence. Both UniFrac metrics utilize information on the phylogenetic diversity of bacterial members when calculating microbiota similarity. PERM ANOVA model \#1 included sample month, host dietary guild, and host family as predictors (in this order) and included all 11 host species. Model 2 was identical to Model 1 , except it included host species in lieu of host family. Model 3 was similar to Model 2, except it was restricted to the Bovid dataset (7 host species). Microbiota similarity and groupings across samples were visualized via Principal Coordinates Analysis (PCoA) plots.

To test for phylosymbiosis, i.e., the congruence between host phylogenetic relatedness and gut microbiota similarity, mean divergence times (mya) were calculated between every pair of host species in R. First, we retrieved 1000 phylogenetic trees that included all species of Artiodactyla and African elephants (Loxodonta Africana) from Upham's et al. (2019) Mammalian supertree [107]. The trees were randomly sampled from the posterior distribution of Upham's supertrees (Mammals birth-death tip-dated DNA-only trees) using the VertLife online resource (http://vertlife.org/). Each tree was pruned to include only the species in this study, and branch lengths (i.e. divergence times between each pair of host species) were extracted using the $\mathrm{R}$ ape package (v.5.4.1) [108]. All 1000 trees showed the same phylogenetic relationships among the study species and matrices of mean divergence times were estimated from those trees. To determine the strength of the phylosymbiosis signal relative to influences attributable to host diet, we conducted partial correlation tests using Spearman correlations with the $R$ ppcor package [109]. These tests correlated i) gut microbiota dissimilarity with host phylogenetic distance (divergence times), while controlling for dietary similarity $(\% \mathrm{C} 4)$, or ii) correlated gut microbiota dissimilarity with dietary dissimilarity, while controlling for host phylogenetic distance.

We visualized the phylosymbiosis findings by plotting gut microbiota similarity $(0-1)$ against host phylogenetic divergence time (mya) in ggplot2. We added a trendline to this plot for plotting purposes; the trendline represented the best fit line of a linear model regressing Bray-Curtis dissimilarity with host phylogenetic distance. We also constructed a consensus phylogeny of our host species and compared it against a dendrogram of gut microbiota dissimilarity, which was calculated using hierarchical clustering with the $\mathrm{R}$ stats package [92] and plotted using the ape package.

\section{Comparisons of Masai Mara and Laikipia herbivores}

In order to compare the gut microbiotas of Masai Mara $\left(1^{\circ} 22^{\prime} 19^{\prime \prime} \mathrm{S}, 34^{\circ} 56^{\prime} 17^{\prime \prime} \mathrm{E}\right)$ herbivores to the gut microbiotas of their conspecifics in Laikipia $\left(0^{\circ} 17^{\prime} 33^{\prime \prime} \mathrm{N}, 36^{\circ}\right.$ $\left.53^{\prime} 55^{\prime \prime} \mathrm{E}\right)$ (> $300 \mathrm{~km}$ from the Masai Mara), we downloaded all publicly available sequences from Kartzinel et al. [22], and combined them with the raw $16 \mathrm{~S}$ rRNA gene sequences from this study (Masai Mara herbivores). The sequences from both studies were then processed together in DADA2. A total of eight herbivore species overlapped between the two studies: African buffalo, domestic cattle, common eland, impala, giraffe, warthog, plains zebra, and African elephant. 96\% of samples from Kartzinel et al. [22] and 81\% of samples from our study were collected during the wet seasons in their respective regions (Table S10), although, in general, Laikipia is more arid than the Masai Mara, with only 300-600 mm precipitation annually [26, 110, 111]. For a list of all samples $(N=305)$, and their associated metadata, see the Availability of data and materials section.

The bioinformatics processing and statistical analyses were performed as described above, with a few exceptions. In DADA2, forward and reverse reads were trimmed to $240 \mathrm{bp}$ and $150 \mathrm{bp}$, respectively, to better account for sequence quality. Up to 2 errors were allowed per forward read and up to 4 errors per reverse read. To identify the strongest predictors of gut microbiota structure, we constructed a PERMANOVA model that included sample month, host geographic region, host dietary guild, and host species as variables (in this order). PCoA ordinations and testing for phylosymbiosis were conducted as described earlier. To visualize gut microbiota compositions between Masai Mara and Laikipia herbivores, a heatmap of the 32 most abundant bacterial ASVs was constructed using R pheatmap. We furthered compared the gut microbiotas of conspecific hosts by conducting Linear discriminant analysis Effect Size (LEfSe) [112] in the Galaxy platform [113] using default parameters. Only ASVs > $0.01 \%$ average relative abundance across samples were included in the dataframe uploaded to Galaxy. ASVs that were enriched in hosts from one geographic region relative to the other were visualized via diverging dot plots in $\mathrm{R}$ with the ggplot2 package.

\section{Abbreviations}

ASV: Amplicon sequence variant; LMM: Linear mixed model; PERM ANOVA: Permutational multivariate analysis of variance;

PCoA: principal coordinates analysis; LEfSe : Linear discriminant analysis effect size; NCBI: National center for biotechnology information; SCFAs: Short chain fatty acids; UIC : University of Illinois at Chicago; DADA2: Divisive amplicon denoising algorithm 2; PD: Faith's phylogenetic diversity; mya: Millions of years ago; IACUC: Institutional animal care and use committee 


\section{Supplementary Information}

The online version contains supplementary material available at https://doi. org/10.1186/s42523-021-00094-4

Additional file 1: Tables S1-S8. Table S1. Previously published dietary \%C4 data for the surveyed herbivores. Table S2. PERMANOVA tests that include sample month, host dietary guild, and host species using the entire dataset (11 sp). Table S3-S6. Multiple-comparison testing of gut microbiota alpha-diversity among host families, species, and dietary guilds. Table S4. Previously published dietary \%C4 data for the surveyed herbivores. Table S7. PERMANOVA tests that included sample month, geographic region, host dietary guild and host species for the combined Masai Mara and Laikipia dataset. Table S8. Partial correlation coefficient statistics regressing microbiota similarity against host phylogenetic relatedness or diet. Table S9. Proportion of ASVs that were differentially enriched in herbivores from one geographic region compared to the other according to LEfSe. Table S10. Sample sizes for each month for the combined Masai Mara and Laikipia dataset (.xlxs 29 KB).

Additional file 2: Figs. S1-S6. Fig. S1-S2. Stacked bar plots showing relative abundances of top bacterial phyla and genera. Fig. S3. Heatmap of the relative abundances of 10 widely-shared bacterial ASVs. Fig. S4. Stacked barplot of the proportion of samples from each host species that were collected each month (for the Masai Mara dataset). Fig. S5. Rarefaction curves of ASV richness for the study samples (.pdf 1.9 MB).

\section{Acknowledgements}

We thank the field assistants and graduate students from the Mara Hyena Project for assisting with fecal sample collection in the field. We also thank the Kenya Wildlife Service, the Kenyan National Commission on Science, Technology and Innovation, the Kenyan National Environmental Management Authority, the Narok County Government, the Naboisho Conservancy, the senior warden of the Masai Mara, the Mara Conservancy, and Brian Heath for allowing us to conduct this research.

\section{Authors' contributions}

K.R.T., K.E.H., and C.A.R. designed the study, C.A.R. and Mara Hyena Project field assistants collected the samples. C.A.R., S.R.B., and K.R.T. analyzed the data. C.A.R., K.R.T, and K.E.H. wrote the manuscript and all authors approved the final version of the manuscript.

\section{Author's information}

Not applicable.

\section{Funding}

This work was supported by funds from NSF grants OISE1853934 and IOS 1755089 to K.E.H. and by the BEACON Center for the Study of Evolution in Action funded as NSF award OIA0939454. The corresponding author (C.A.R.) was also supported by a Graduate Research Fellowship from NSF, a Predoctoral Fellowship from the Ford Foundation, and a summer fellowship awarded by the Ecology, Evolution, and Behavior program at MSU.

\section{Availability of data and materials}

The 16S rRNA gene sequence data from this study were deposited in NCBI's Sequence Read Archive under BioProject PRJNA656793 and accession numbers SAMN15803511- SAMN15803691. Sample metadata, data output by DADA2 (ASV table \& ASV taxonomic classifications), data obtained from LEfSe, and R scripts for all analyses and figures included in this manuscript are available on Github (https://github.com/rojascon/Rojas_et_al_2020_ African_herbivores_gut_microbiome).

\section{Declarations}

\section{Ethics approval and consent to participate}

Our research and procedures were most recently approved on January 8 , 2020 (MSU IACUC approval no. PROTO201900126) and comply with the ethical standards of Michigan State University and Kenya.

\section{Consent for publication}

Not applicable.

\section{Competing interests}

The authors declare that there are no competing interests.

\section{Author details}

${ }^{1}$ Department of Integrative Biology, Michigan State University, East Lansing, MI, USA. ${ }^{2}$ Ecology, Evolution, and Behavior Program, Michigan State University, East Lansing, MI, USA. ${ }^{3}$ BEACON Center for the Study of Evolution in Action, Michigan State University, East Lansing, MI, USA. ${ }^{4}$ Departament of Botany, Institute of Biology, Universidad Nacional Autónoma de México, Mexico City, MX, Mexico. ${ }^{5}$ Department of Biochemistry, Microbiology and Immunology, Wayne State University School of Medicine, Detroit, MI, USA.

Received: 17 September 2020 Accepted: 4 April 2021

Published online: 23 April 2021

\section{References}

1. Jovel J, Dieleman LA, Kao D, Mason AL, Wine E. The human gut microbiome in health and disease. Metagenomics. 2018:197-213. https:// doi.org/10.1016/B978-0-08-102268-9.00010-0.

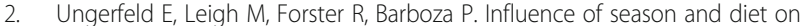
Fiber digestion and bacterial community structure in the rumen of muskoxen (Ovibos moschatus). Microorganisms. 2018;6(3):89. https://doi. org/10.3390/microorganisms6030089.

3. Greene LK, McKenney EA, O'Connell TM, Drea CM. The critical role of dietary foliage in maintaining the gut microbiome and metabolome of folivorous sifakas. Sci Rep. 2018;8:1-13.

4. den Besten G, van Eunen K, Groen A, Venema K, Reijngoud D, Bakker B. The role of short-chain fatty acids in the between diet, gut microbiota, and host energy metabolism. J Lipid Res. 2013;54(9):2325-40. https://doi.org/10.1194/ jlr.R036012.

5. Park W. Gut microbiomes and their metabolites shape human and animal health. J Microbiol. 2018;56(3):151-3. https://doi.org/10.1007/s12275-0180577-8.

6. Münger E, Montiel-Castro AJ, Langhans W, Pacheco-López G. Reciprocal interactions between gut microbiota and host social behavior. Front Integr Neurosci. 2018;12:1-14. https://doi.org/10.3389/fnint.2018.00021.

7. Pennisi E. Gut bacteria linked to mental well-being and depression. Science (80- ). 2019;363:569. https://doi.org/10.1126/science.363.6427.569.

8. Li J, Zhan S, Liu X, Lin Q, Jiang J, Li X. Divergence of fecal microbiota and their associations with host phylogeny in Cervinae. Front Microbiol. 2018;9: 1-11. https://doi.org/10.3389/fmicb.2018.01823.

9. Amato KR, Sanders J, Song SJ, Nute M, Metcalf JL, Thompson LR, et al. Evolutionary trends in host physiology outweigh dietary niche in structuring primate gut microbiomes. ISME J. 2019;13(3):576-87. https://doi.org/10.103 8/s41396-018-0175-0.

10. Nishida $\mathrm{AH}$, Ochman $\mathrm{H}$. Rates of gut microbiome divergence in mammals. Mol Ecol. 2018;27(8):1884-97. https://doi.org/10.1111/mec.14473.

11. Youngblut ND, Reischer GH, Walters W, Schuster N, Walzer C, Stalder G, et al. Host diet and evolutionary history explain different aspects of gut microbiome diversity among vertebrate clades. Nat Commun. 2019;10(1):115. https://doi.org/10.1038/s41467-019-10191-3.

12. Moeller AH, Suzuki TA, Lin D, Lacey EA, Wasser SK, Nachman MW. Dispersal limitation promotes the diversification of the mammalian gut microbiota. PNAS. 2017:114(52):13768-73. https://doi.org/10.1073/pnas.1700122114.

13. Kohl KD. Ecological and evolutionary mechanisms underlying patterns of phylosymbiosis in host-associated microbial communities. Philos Trans R Soc B Biol Sci. 2020;375(1798):20190251. https://doi.org/10.1098/rstb.2019.02 51.

14. Lim SJ, Bordenstein SR. An introduction to phylosymbiosis. Proc R Soc B Biol Sci. 2020;287:1-10

15. Brooks AW, Kohl KD, Brucker RM, Van Opstal EJ, Bordenstein SR. Phylosymbiosis: relationships and functional effects of microbial communities across host evolutionary history. PLoS Biol. 2016;14(11):1-19. https://doi.org/10.1371/journal.pbio.2000225

16. Watson SE, Hauffe HC, Bull M, McKinney MA, Atwood TA, Perkins SE. Global change-driven use of onshore habitat impacts polar bear faecal microbiota. ISME J. 2019;13(12):2916-26. https://doi.org/10.1038/s41396-019-0480-2.

17. Angelakis E, Yasir M, Bachar D, Azhar El, Lagier JC, Bibi F, et al. Gut microbiome and dietary patterns in different Saudi populations and monkeys. Sci Rep. 2016;6(1):1-9. https://doi.org/10.1038/srep32191. 
18. Goertz S, De Menezes AB, Birtles RJ, Id JF, Lowe E, Maccoll ADC, et al. Geographical location influences the composition of the gut microbiota in wild house mice (Mus musculus domesticus) at a fine spatial scale. PLoS One. 2019:14:1-16.

19. Orkin JD, Campos FA, Guadamuz A, Melin AD, Myers MS, Hernandez SEC. Seasonality of the gut microbiota of free-ranging white-faced capuchins in a tropical dry forest. ISME J. 2019;13(1):183-96. https://doi.org/10.1038/s413 96-018-0256-0.

20. Knowles SCL, Eccles RM, Baltrūnaite L. Species identity dominates over environment in shaping the microbiota of small mammals. Ecol Lett. 2019; 22(5):826-37. https://doi.org/10.1111/ele.13240.

21. Kohl KD, Varner J, Wilkening $J$, Dearing MD. Gut microbial communities of American pikas (Ochotona princeps): evidence for phylosymbiosis and adaptations to novel diets. J Anim Ecol. 2018;87(2):323-30. https://doi.org/1 $0.1111 / 1365-2656.12692$

22. Kartzinel TR, Hsing JC, Musili PM, Brown BRP, Pringle RM. Covariation of diet and gut microbiome in African megafauna. PNAS. 2019;116:1-6.

23. Ottichilo WK, De Leeuw J, Skidmore AK, Prins HHT, Said MY. Population trends of large non-migratory wild herbivores and livestock in the Masai Mara ecosystem, Kenya, between 1977 and 1997. Afr J Ecol. 2000;38(3):20216. https://doi.org/10.1046/j.1365-2028.2000.00242.x.

24. Goheen JR, Augustine DJ, Veblen KE, Kimuyu DM, Palmer TM, Porensky LM, et al. Conservation lessons from large-mammal manipulations in east African savannas: the KLEE, UHURU, and GLADE experiments. Ann N Y Acad Sci. 2018;1429(1):31-49. https://doi.org/10.1111/nyas.13848.

25. Veldhuis MP, Ritchie ME, Ogutu JO, Morrison TA, Beale CM, Estes AB, et al. Cross-boundary human impacts compromise the Serengeti-Mara ecosystem. Science (80- ). 2019;363:1424-8. https://doi.org/10.1126/science.aav0564.

26. Augustine DJ. Response of native ungulates to drought in semi-arid Kenyan rangeland. Afr J Ecol. 2010;48(4):1009-20. https://doi.org/10.1111/j.1365-202 8.2010.01207.x.

27. Broten MD, Said M. Population Trends of Ungulates in and around Kenya's Masai Mara Reserve. In: Serengeti II: Dynamics, Management, and Conservation of an Ecosystem. 1995. p. 169-93.

28. Cerling TE, Harris JM, Passey BH. Diets of east African Bovidae based on stable isotope analysis. J Mammal. 2003;84(2):456-70. https://doi.org/10.1 644/1545-1542(2003)084<0456:DOEABB>2.0.CO;2.

29. Kartzinel TR, Chen PA, Coverdale TC, Erickson DL, Kress WJ, Kuzmina ML, et al. DNA metabarcoding illuminates dietary niche partitioning by African large herbivores. PNAS. 2015;112(26):8019-24. https://doi.org/10.1073/pnas.1 503283112.

30. Codron D, Codron J, Lee-Thorp JA, Sponheimer M, De Ruiter D, Sealy J, et al. Diets of savanna ungulates from stable carbon isotope composition of faeces. J Zool. 2007;273(1):21-9. https://doi.org/10.1111/j.1469-7998.2007.002 92.x.

31. Codron J, Lee-Thorp JA, Sponheimer M, Codron D, Grant R, De Ruiter D. Elephant (Loxodonta Africana) diets in Kruger National Park, South Africa: spatial and landscape differences. J Mammal. 2006;87(1):27-34. https://doi. org/10.1644/05-MAMM-A-017R1.1.

32. Scheelings TF, Moore RJ, Van TTH, Klaassen M, Reina RD. Microbial symbiosis and coevolution of an entire clade of ancient vertebrates: the gut microbiota of sea turtles and its relationship to their phylogenetic history. Anim Microbiome. 2020;2:2-12.

33. Tinker KA, Ottesen EA. Phylosymbiosis across deeply diverging lineages in omnivorous cockroaches. Appl Environ Microbiol. 2020;86(7):e02513-9. https://doi.org/10.1128/AEM.02513-19.

34. Schmidt E, Mykytczuk N, Schulte-Hostedde Al. Effects of the captive and wild environment on diversity of the gut microbiome of deer mice (Peromyscus maniculatus). ISME J. 2019;13(5):1293-305. https://doi.org/10.1 038/s41396-019-0345-8.

35. Perofsky AC, Lewis RJ, Meyers LA. Terrestriality and bacterial transfer: a comparative study of gut microbiomes in sympatric Malagasy mammals. ISME J. 2019;13(1):50-63. https://doi.org/10.1038/s41396-018-0251-5.

36. Greene LK, Clayton JB, Rothman RS, Semel BP, Semel MA, Gillespie TR, et al. Local habitat, not phylogenetic relatedness, predicts gut microbiota better within folivorous than frugivorous lemur lineages. Biol Lett. 2019;15:5-11.

37. Gogarten JF, Davies TJ, Benjamino J, Gogarten JP, Graf J, Mielke A, et al. Factors influencing bacterial microbiome composition in a wild non-human primate community in Taï National Park, Côte d'Ivoire. ISME J. 2018;12(10): 2559-74. https://doi.org/10.1038/s41396-018-0166-1.
38. Amato KR, Mallott EK, Mcdonald D, Dominy NJ, Goldberg T, Lambert JE, et al. Convergence of human and Old World monkey gut microbiomes demonstrates the importance of human ecology over phylogeny. Genome Biol. 2019;20(1):201. https://doi.org/10.1186/s13059-019-1807-z.

39. Groussin M, Mazel F, Sanders JG, Smillie CS, Lavergne S, Thuiller W, et al. Unraveling the processes shaping mammalian gut microbiomes over evolutionary time. Nat Commun. 2017;8(1):14319. https://doi.org/10.1038/ ncomms14319.

40. Groussin M, Mazel F, Alm EJ. Co-evolution and co-speciation of host-gut Bacteria systems. Cell Host Microbe. 2020;28(1):12-22. https://doi.org/10.101 6/j.chom.2020.06.013.

41. Gerardo NM, Hoang KL, Stoy KS. Evolution of animal Immunity in the light of beneficial symbioses. Philos Trans R Soc B2. 2020;375:20190601.

42. Popkes M, Valenzano DR. Microbiota-host interactions shape ageing dynamics. Philos Trans R Soc B2. 2020;375:20190596.

43. Perofsky AC, Lewis RJ, Abondano LA, Difiore A, Meyers LA. Hierarchical social networks shape gut microbial composition in wild verreaux's sifaka. Proc R Soc B Biol Sci. 2017;284:1-10.

44. Raulo A, Ruokolainen L, Lane A, Amato K, Knight R, Leigh S, et al. Social behaviour and gut microbiota in red-bellied lemurs ( Eulemur rubriventer ): in search of the role of immunity in the evolution of sociality. J Anim Ecol. 2017;87(2):1-12. https://doi.org/10.1111/1365-2656.12781.

45. Opie C, Atkinson QD, Shultz S. The evolutionary history of primate mating systems. Commun Integr Biol. 2012;5(5):458-61. https://doi.org/10.4161/cib.2 0821.

46. Shultz S, Opie C, Atkinson QD. Stepwise evolution of stable sociality in primates. Nature. 2011;479(7372):219-22. https://doi.org/10.1038/nature1 0601.

47. Grieneisen LE, Charpentier MJE, Alberts SC, Blekhman R, Bradburd G, Tung J, et al. Genes, geology and germs: gut microbiota across a primate hybrid zone are explained by site soil properties, not host species. Proc R Soc B Biol Sci. 2019:286:1-9.

48. Muegge BD, Kuczynski J, Kinghts D, Clemente JC, Fontana L, Henrissat B, et al. Diet drives convergence in gut microbiome functions across mammalian phylogeny and within humans. Science (80- ). 2017;332:970-4.

49. Clemens ET, Maloiy GMO. Digestive physiology of east African wild ruminants. Comp Biochem Physiol Part A Physiol. 1983;76(2):319-33. https:// doi.org/10.1016/0300-9629(83)90333-X.

50. Illius AW, Gordon IJ. The functional significance of the browser-grazer dichotomy in African ruminants. Oecologia. 1994;98:167-75.

51. Pérez-Barbería JF, Gordon IJ, Illius AW. Phylogenetic analysis of stomach adaptation in digestive strategies in African ruminants. Oecologia. 2001; 129(4):498-508. https://doi.org/10.1007/s004420100768.

52. Ley RE, Hamady M, Lozupone C, Turnbaugh PJ, Ramey RR, Bircher JS, et al. Evolution of mammals and their gut microbes. Science (80- ). 2008;320:1647.

53. McKenzie VJ, Song SJ, Delsuc F, Prest TL, Oliverio AM, Korpita TM, et al. The effects of captivity on the mammalian gut microbiome. Integr Comp Biol. 2017;57(4):690-704. https://doi.org/10.1093/icb/icx090.

54. Phillips CD, Phelan G, Dowd SE, McDonough MM, Ferguson AW, Delton Hanson J, et al. Microbiome analysis among bats describes influences of host phylogeny, life history, physiology and geography. Mol Ecol. 2012; 21(11):2617-27. https://doi.org/10.1111/j.1365-294X.2012.05568.x.

55. Godon JJ, Arulazhagan P, Steyer JP, Hamelin J. Vertebrate bacterial gut diversity: size also matters. BMC Ecol. 2016;16:1-9.

56. Liu $P$, Cheng A, Huang $S$, Lu H, Oshida T. Body-size scaling is related to gut microbial diversity, metabolism and dietary niche of arboreal folivorous flying squirrels. Sci Rep. 2020:1-12. https://doi.org/10.1038/s41598-020-64801-y.

57. Budd K, Gunn JC, Eggert LS, Finch T, Klymus K, Sitati N. Effects of diet, habitat, and phylogeny on the fecal microbiome of wild African savanna (Loxodonta africana) and forest elephants (L . cyclotis). Ecol Evol. 2020; 10(12):1-14

58. Metcalf JL, Song SJ, Morton JT, Weiss S, Seguin-Orlando A, Joly F, et al. Evaluating the impact of domestication and captivity on the horse gut microbiome. Sci Rep. 2017;7(1):15497. https://doi.org/10.1038/s41598-017-1 5375-9.

59. Miller EA, Livermore J, Alberts SC, Tung J, Archie EA. Ovarian cycling and reproductive state shape the vaginal microbiota in wild baboons. Microbiome. 2017:5:1-14.

60. Moeller AHA, Foerster S, Wilson ML, Pusey AE, Hahn BH, Ochman H. Social behavior shapes the chimpanzee pan-microbiome. Sci Adv. 2016;2(1): e1500997. https://doi.org/10.1126/sciadv.1500997. 
61. Johnson KVA. Gut microbiome composition and diversity are related to human personality traits. Hum Microbiome J. 2020;15 December 2019: 100069. https://doi.org/10.1016/j.humic.2019.100069.

62. Shipley LA. Grazer s and browsers: how digestive morphology affects diet selection. In: Launchbaugh KL, Sanders KD, Mosley JC, editors. Grazing Behavior of Livestock and Wildlife. Moscow: Univ. of Idaho; 1999. p. 20-7.

63. Venter JA, Vermeulen MM, Brooke CF. Feeding ecology of large browsing and grazing herbivores. In: Gordon IJ, Prins HHT, editors. The ecology of browsing and grazing II. Cham: Springer International Publishing; 2019. p. 127-53. https://doi.org/10.1007/978-3-030-25865-8_5.

64. Hofmann RR. Evolutionary steps of ecophysiological adaptation and diversification of ruminants: a comparative view of their digestive system. Oecologia. 1989;78(4):443-57. https://doi.org/10.1007/BF00378733.

65. Spencer LM. Morphological correlates of dietary resource partitioning in the African Bovidae. J Mammal. 1995;76(2):448-71. https://doi.org/10.2307/1382355.

66. Henderson G, Cox F, Ganesh S, Jonker A, Young W, Janssen PH, et al. Rumen microbial community composition varies with diet and host, but a core microbiome is found across a wide geographical range. Sci Rep. 2015; 5(1):14567. https://doi.org/10.1038/srep14567.

67. Edwards JE, Shetty SA, Van Den Berg P, Burden F, Van Doorn DA, Pellikaan WF, et al. Multi-kingdom characterization of the core equine fecal microbiota based on multiple equine (sub) species. Anim Microbiome. 2020; 2:1-16.

68. Ilmberger N, Güllert S, Dannenberg J, Rabausch U, Torres J, Wemheuer B, et al. A comparative metagenome survey of the fecal microbiota of a breast-and a plant-fed asian elephant reveals an unexpectedly high diversity of glycoside hydrolase family enzymes. PLoS One. 2014;9:1-12.

69. Roggenbuck M, Sauer C, Poulsen M, Bertelsen MF, Sørensen SJ. The giraffe (Giraffa camelopardalis) rumen microbiome. FEMS Microbiol Ecol. 2014;90(1): 237-46. https://doi.org/10.1111/1574-6941.12402.

70. Tan SC, Chong CW, Yap IKS, Thong KL, Teh CSJ. Comparative assessment of faecal microbial composition and metabonome of swine, farmers and human control. Sci Rep. 2020;10:1-13.

71. Zeng B, Zhang S, Xu H, Kong F, Yu X, Wang P, et al. Gut microbiota of Tibetans and Tibetan pigs varies between high and low altitude environments. Microbiol Res. 2020;235 November 2019:126447. https://doi. org/10.1016/j.micres.2020.126447.

72. Biddle A, Stewart L, Blanchard J, Leschine S. Untangling the genetic basis of fibrolytic specialization by Lachnospiraceae and Ruminococcaceae in diverse gut communities. Diversity. 2013;5(3):627-40. https://doi.org/10.33 90/d5030627.

73. Flint HJ, Scott KP, Duncan SH, Louis P, Forano E. Microbial degradation of complex carbohydrates in the gut. Gut Microbes. 2012;3(4):289-306.

74. Kohl KD, Denise DM. The woodrat gut microbiota as an experimental system for understanding microbial metabolism of dietary toxins. Front Microbiol. 2016;7:1-9.

75. Coordinators NR. Database resources of the National Center for biotechnology information. Nucleic Acids Res. 2018;46(D1):D8-D13. https:// doi.org/10.1093/nar/gkx1095.

76. Shorrocks B. The biology of African savannas. New York: Oxford University Press; 2007. https://doi.org/10.1093/acprof:oso/9780198570660.001.0001.

77. Wright ADG, Auckland CH, Lynn DH. Molecular diversity of methanogens in feedlot cattle from Ontario and Prince Edward Island, Canada. Appl Environ Microbiol. 2007;73(13):4206-10. https://doi.org/1 0.1128/AEM.00103-07.

78. Daniel H, Gholami AM, Berry D, Desmarchelier C, Hahne H, Loh G, et al. High-fat diet alters gut microbiota physiology in mice. ISME J. 2014;8(2): 295-308. https://doi.org/10.1038/ismej.2013.155.

79. Liu J, Pu YY, Xie Q, Wang JK, Liu JX. Pectin induces an in vitro rumen microbial population shift attributed to the Pectinolytic Treponema group. Curr Microbiol. 2014;70(1):67-74. https://doi.org/10.1007/s00284014-0672-y.

80. Xie X, Yang C, Guan LL, Wang J, Xue M, Liu JX. Persistence of cellulolytic bacteria fibrobacter and treponema after short-term corn stover-based dietary intervention reveals the potential to improve rumen fibrolytic function. Front Microbiol. 2018;9 JUN:1363. doi:https://doi.org/10.3389/ fmicb.2018.01363.

81. Milani C, Alessandri G, Mancabelli L, Mangifesta M, Lugli GA, Viappiani A, et al. Multi-omics approaches to decipher the impact of diet and host physiology on the mammalian gut microbiome. Appl Environ Microbiol. 2020;86:1-21.
82. Kennedy K, Hall MW, Lynch MDJ, Moreno-Hagelsieb G, Neufeld JD. Evaluating bias of Illumina-based bacterial 16S rRNA gene profiles. Appl Environ Microbiol. 2014;80(18):5717-22. https://doi.org/10.1128/AEM.014 51-14.

83. De La Cuesta-Zuluaga J, Escobar JS. Considerations for optimizing microbiome analysis using a marker gene. Front Nutr. 2016;3:26. https://doi. org/10.3389/fnut.2016.00026

84. Mallott EK, Malhi RS, Amato KR. Assessing the comparability of different DNA extraction and amplification methods in gut microbial community profiling. Access Microbiol. 2019;1(7):1-15.

85. Green DS, Zipkin EF, Incorvaia DC, Holekamp KE. Long-term ecological changes influence herbivore diversity and abundance inside a protected area in the Mara-Serengeti ecosystem. Glob Ecol Conserv. 2019;20:e00697. https://doi.org/10.1016/j.gecco.2019.e00697.

86. Kingdon J. East African mammals: volume I. Chicago: The University of Chicago Press; 1984

87. Kingdon J. East African mammals: volume IIIC. Chicago: The University of Chicago Press; 1982.

88. Kingdon J. East African mammals: volume IIID. Chicago: The University of Chicago Press; 1982

89. Kingdon J. East African mammals: volume IIIB. Chicago: The University of Chicago Press; 1979.

90. Caporaso JG, Lauber CL, Walters WA, Berg-Lyons D, Huntley J, Fierer N, et al. Ultra-high-throughput microbial community analysis on the Illumina HiSeq and MiSeq platforms. ISME J. 2012;6(8):1621-4. https://doi.org/10.1038/ ismej.2012.8.

91. Kozich JJ, Westcott SL, Baxter NT, Highlander SK, Schloss PD. Development of a dual-index sequencing strategy and Curation pipeline for analyzing amplicon sequence data on the MiSeq Illumina sequencing platform. Appl Environ Microbiol. 2013;79(17):5112-20. https://doi.org/10.1128/AEM.01043-13.

92. R Core Team. R: a language and environment for statistical computing. Vienna, Austria: R Foundation for Statistical Computing; 2019. https://www. gbif.org/tool/81287/r-a-language-and-environment-for-statistical-computing. Accessed 28 Aug 2019

93. Callahan BJ, McMurdie PJ, Rosen MJ, Han AW, Johnson AJA, Holmes SP. DADA2: high-resolution sample inference from Illumina amplicon data. Nat Methods. 2016;13(7):581-3. https://doi.org/10.1038/nmeth.3869.

94. Quast C, Pruesse E, Yilmaz P, Gerken J, Schweer T, Yarza P, et al. The SILVA ribosomal RNA gene database project: improved data processing and webbased tools. Nucleic Acids Res. 2013;41(D1):D590-6. https://doi.org/10.1093/ nar/gks1219.

95. Wickham H. ggplot2: elegant graphics for Dat analysis. Springer-Verlag New York: New York, NY; 2009. https://doi.org/10.1007/978-0-387-98141-3.

96. Kolde R. Pheatmap: Pretty Heatmaps. R package version 1.0.12. 2019::1-8. https://cran.r-project.org/package=pheatmap.

97. De Cáceres M, Legendre P. Associations between species and groups of sites: indices and statistical inference. Ecology. 2009;90(12):3566-74. https:// doi.org/10.1890/08-1823.1

98. Schloss PD, Westcott SL, Ryabin T, Hall JR, Hartmann M, Hollister EB, et al. Introducing mothur: open-source, platform-independent, communitysupported software for describing and comparing microbial communities. Appl Environ Microbiol. 2009;75(23):7537-41. https://doi.org/10.1128/AEM.01 541-09.

99. Jin L, Wu D, Li C, Zhang A, Xiong Y, Wei R, et al. Bamboo nutrients and microbiome affect gut microbiome of giant panda. Symbio. 2020;80(3):293304. https://doi.org/10.1007/s13199-020-00673-0.

100. Bo T-B, Zhang X-Y, Wen J, Deng K, Qin X-W, Wang D-H. The microbiota-gutbrain interaction in regulating host metabolic adaptation to cold in male Brandt's voles (Lasiopodomys brandtii). ISME J. 2019;13(12):3037-53. https:// doi.org/10.1038/s41396-019-0492-y.

101. McMurdie PJ, Holmes S. Phyloseq: an R package for reproducible interactive analysis and graphics of microbiome census data. PLoS One. 2013;8:1-11.

102. Schliep KP. Phangorn: phylogenetic analysis in R. Bioinformatics. 2011;27(4): 592-3. https://doi.org/10.1093/bioinformatics/bta706.

103. Kembel SW, Cowan PD, Helmus MR, Cornwell WK, Morlon H, Ackerly DD, et al. Picante: $\mathrm{R}$ tools for integrating phylogenies and ecology. Bioinformatics. 2010;26(11):1463-4. https://doi.org/10.1093/bioinformatics/btq166.

104. Bates D, Mächler M, Bolker B, Walker S. Fitting linear mixed-effects models using Ime4. J Stat Softw. 2015;67:1-48. https://doi.org/10.18637/jss.v067.i01

105. Fox J, Weisberg S, Fox J. An R companion to applied regression. Thousand Oaks, CA: Sage; 2011. 
106. Oksanen J, Blanchet F, Friendly M, Kindt R, Legendre P, McGlinn D, et al. Vegan: Community Ecology Package. R Packag version 24-6. 2018. https:// cran.r-project.org/web/packages/vegan/index.html. Accessed 28 Aug 2019.

107. Upham N, Esselstyn J, Jetz W. Inferring the mammal tree: species-level sets of phylogenies for questions in ecology, evolution, and conservation. PLoS Biol. 2019;17(12):e3000494. https://doi.org/10.1371/journal.pbio.3000494.

108. Paradis E, Claude J, Strimmer K. APE: analyses of phylogenetics and evolution in R language. Bioinformatics. 2004;20(2):289-90. https://doi.org/1 0.1093/bioinformatics/btg412.

109. Kim S. Ppcor: an R package for a fast calculation to semi-partial correlation coefficients. Commun Stat Appl Methods. 2015;22(6):665-74. https://doi. org/10.5351/CSAM.2015.22.6.665.

110. Ochieng EO. Characterizing the spatial distributions of elephants in Mpala, Kenya. 2015.

111. Kartzinel TR, Pringle RM. Multiple dimensions of dietary diversity in large mammalian herbivores. J Anim Ecol. 2020;89(6):1-15.

112. Segata N, Izard J, Waldron L, Gevers D, Miropolsky L, Garrett WS, et al. Metagenomic biomarker discovery and explanation. Genome Biol. 2011; 12(6):R60. https://doi.org/10.1186/gb-2011-12-6-r60.

113. Afgan E, Baker D, Batut B, van den Beek M, Bouvier D, Čech M, et al. The galaxy platform for accessible, reproducible and collaborative biomedical analyses: 2018 update. Nucleic Acids Res. 2018;46(W1):W537-44. https://doi. org/10.1093/nar/gky379.

\section{Publisher's Note}

Springer Nature remains neutral with regard to jurisdictional claims in published maps and institutional affiliations.

Ready to submit your research? Choose BMC and benefit from:

- fast, convenient online submission

- thorough peer review by experienced researchers in your field

- rapid publication on acceptance

- support for research data, including large and complex data types

- gold Open Access which fosters wider collaboration and increased citations

- maximum visibility for your research: over $100 \mathrm{M}$ website views per year

At BMC, research is always in progress.

Learn more biomedcentral.com/submissions 